\title{
Water Diffusion Into UV Inscripted Long Period Grating in Microstructured Polymer Fiber
}

\author{
D. Sáez-Rodríguez, J. L. Cruz, I. Johnson, D. J. Webb, M. C. J. Large, and A. Argyros
}

\begin{abstract}
A long period grating was photoinscribed step-bystep in microstructured poly(methyl methacrylate) fiber for the first time using a continuous wave $\mathrm{HeCd}$ laser at $325 \mathrm{~nm}$, irradiating the fiber with a power of $1 \mathrm{~mW}$. The grating had a length of $2 \mathrm{~cm}$ and a period of $1 \mathrm{~mm}$. A series of cladding mode coupling resonances were observed throughout the spectral region studied of 600 to $1100 \mathrm{~nm}$. The resonance wavelengths were shown to be sensitive to the diffusion of water into the fiber.
\end{abstract}

Index Terms-Humidity, long period grating, microstructured polymer optical fiber (mPOF), optical fiber sensor, photonic crystal fiber (PCF).

\section{INTRODUCTION}

$\mathbf{L}$ ONG PERIOD GRATINGs (LPGs) have been studied for many years in applications such as band rejection filters [1] and sensors of temperature, strain, refractive index (RI), and curvature [2]-[4]. Application of LPGs for relative humidity measurement require to recoat the fiber with an hygroscopic overlayer since cladding modes have negligible interaction with water vapor [5], LPGs in microstructured fiber may offer a new approach for humidity measurement [6] but it can be a difficult task to keep air flowing through the fiber holes. Optical fiber have also been used as water level sensors by measurement of the buoyant force acting on the fiber holder [7] or by direct interaction of the liquid with the guided mode in polished fibers [8], these techniques can be straightforward adapted to Bragg or long period gratings. Recently researchers have begun to investigate the recording of these gratings in polymeric optical fibers [9]. The motivation here is to be able to utilize the different material properties of polymers compared to the traditional silica fiber material. As an example relevant to strain monitoring applications, polymeric fiber can have a much smaller Young's modulus and much higher failure strain than silica fiber [10]. Unlike silica fibers, polymer fibers can absorb water which modifies the properties of the waveguide and therefore have potential applications as distributed water level sensors; moreover, water

Manuscript received September 07, 2009; revised December 04, 2009; accepted February 03, 2010. Date of current version May 19, 2010. This work was supported in part by European Union (EU) FP7 under Project PHOSFOS and in part by the Ministerio de Educació n y Ciencia Español under Grant TEC 2008-05490. The associate editor coordinating the review of this paper and approving it for publication was Dr. Cristiano Cordeiro.

D. Sáez-Rodríguez and J. L. Cruz are with the Dipartimento de Fisica Aplicada y Electromagnetismo, Universidad de Valencia, Burjassot 46100, Valencia, Spain (e-mail: david.saez@uv.es).

I. Johnson and D. J. Webb are with the Photonics Research Group, Aston University, Birmingham, B4 7ET, U.K.

M. C. J. Large and A. Argyros are with the School of Physics, University of Sydney, NSW 2006, Sydney, Australia.

Digital Object Identifier 10.1109/JSEN.2010.2042952 diffusion needs to be investigated in order to prevent unwanted effects of vapor condensation in other sensing applications of polymer optical fibers. Candidate LPGs have previously been produced in microstructured polymer optical fiber (mPOF) by mechanical deformation coupled with heating [9]. In this paper, we describe for the first time to our knowledge, the UV photoinscription of LPGs in mPOF and study the sensitivity to water absorption.

\section{THEORY}

The LPG is a periodic modulation of the effective refractive index of the fiber along of the direction of propagation of the light. It produces attenuation bands at different wavelengths due to coupling from the core mode to various cladding modes. The position of the bands is determined by the resonance condition

$$
\lambda_{\text {res }}=\Lambda \cdot \Delta n
$$

where $\lambda_{\text {res }}$ is the resonant wavelength, $\Lambda$ is the period of grating and $\Delta_{n}=n_{\mathrm{co}}-n_{\mathrm{cl}}$ is the difference in the effective refractive indices of the core and cladding modes.

The sensitivity of the resonance wavelength to a particular measurand, $X$, may be obtained by differentiating (1) to give

$$
\frac{\partial \lambda_{\text {res }}}{\partial X}=\gamma \cdot \lambda_{\text {res }} \cdot\left(\frac{1}{\Lambda} \frac{\partial \Lambda}{\partial X}+\frac{1}{\Delta n} \frac{\partial \Delta n}{\partial X}\right)
$$

where $\gamma$ is a general sensitivity parameter for the fiber [11] given by $(1 / \Delta n) \cdot\left(d \lambda_{\text {res }} / d \Lambda\right)$.

\section{UV INSCRIPTION}

The fiber used in this work is made of poly (methyl methacrylate) (PMMA) with a refractive index of around 1.49. The outside diameter of the fiber was $250 \mu \mathrm{m}$ while the fiber core was elliptical having a large diameter of $7.6 \mu \mathrm{m}$ and small diameter of $4.2 \mu \mathrm{m}$. The core was surrounded by 56 air holes with $0.72 \mu \mathrm{m}$ diameter and a separation of $2.59 \mu \mathrm{m}$, which gave a ratio of hole diameter to spacing of $d / \Lambda=0.28$. As a consequence of the elliptical core, the fiber has a non-negligible birefringence.

Before writing the gratings, the ends of the $\mathrm{mPOF}$ fiber were spliced to two single mode silica fibers. The mPOF fiber was cleaved on a hot plate at $80^{\circ} \mathrm{C}$ in order to achieve a flat front surface and to prevent the air holes from collapse. The fibers to be spliced were mounted on XYZ stages in order to align their cores, the alignment being optimized by monitoring the light transmitted through the junction. The aligned fibers were fixed with Loctite 3525 glue cured during 10 min of exposure to a UV lamp having $0.5 \mathrm{~W}$ of nominal power. Finally, the splices were sleeved into silica tubes of $2.75 \mathrm{~mm}$ outer diameter in order to 


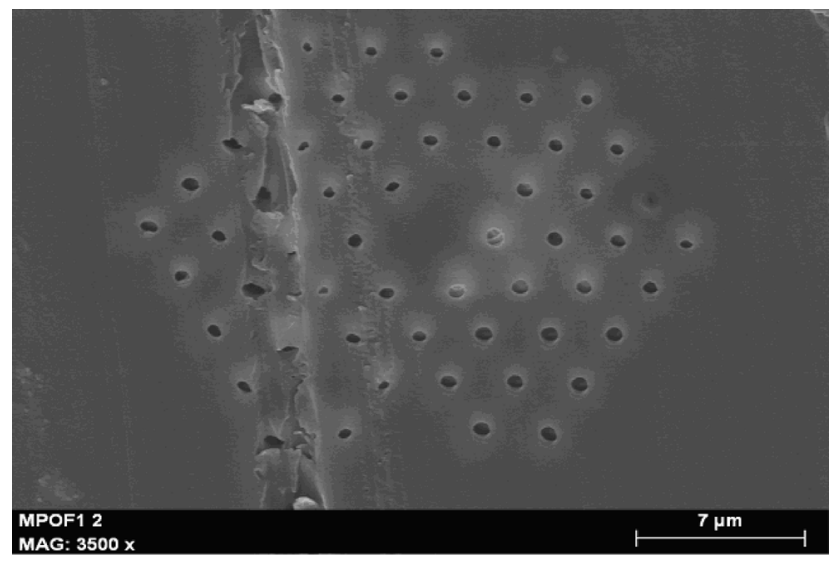

Fig. 1. Electron microscope photograph of a transversal cut of the mPOF.

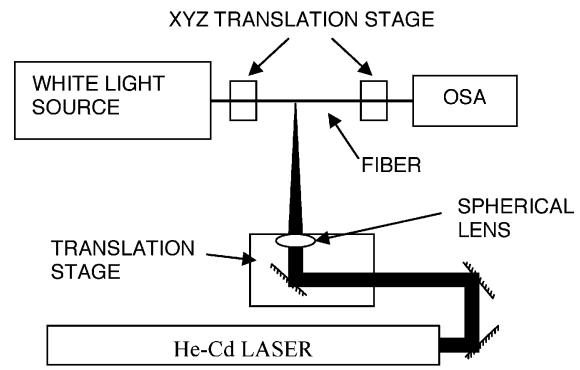

Fig. 2. Experimental arrangement.

strengthen them. The insertion loss of the spliced fiber was measured to be $14 \mathrm{~dB}$ in fibers used in this experiment. Fig. 1 shows a SEM photograph of the cross section of the cleaved fiber, the elliptical core can be observed as well as a scratch made by the cleaver. Besides the fiber compatibility, splice loss to silica fiber is dependent on the quality of the fiber cut, the fiber alignment and the gluing process. Splice loss has been reduced to $6 \mathrm{~dB}$ in the latest experiments by optimizing the UV curing conditions and the alignment of the fibers to be spliced. Further improvement could be achieved by optimizing the cutting technique, namely the kind of blade used to cleave the fiber.

The grating was written by irradiating the fiber with a continuous wave, $1.2 \mathrm{~mm}$ beam diameter $\mathrm{He}-\mathrm{Cd}$ laser at a wavelength of $325 \mathrm{~nm}$. The laser delivered a maximum power of $30 \mathrm{~mW}$ and was focused onto the fiber by a spherical lens of $20 \mathrm{~cm}$ focal length. The $\mathrm{mPOF}$ was placed on two XYZ translation stages so that it could be aligned with the laser beam. LPGs were written using step-by-step scanning of the fiber past the laser beam and shutting the beam off each time the fiber is moved. The translation stage to scan the laser beam has a precision of $10 \mu \mathrm{m}$. The experimental setup is shown in Fig. 2.

The power delivered by this laser is sufficient to melt the polymer when the beam is focused down. After several attempts irradiating in different conditions and measuring the fiber transmission we concluded that, to prevent holes from collapsing and to reach optimum index change in the fiber, the laser had to be operated at $1 \mathrm{~mW}$ output power and the beam had to be focused over the writing point for a total exposure time of $6 \mathrm{~s}$. The basic mechanism for grating inscription is the refractive index increase due to an increase in density resulting from polymer-

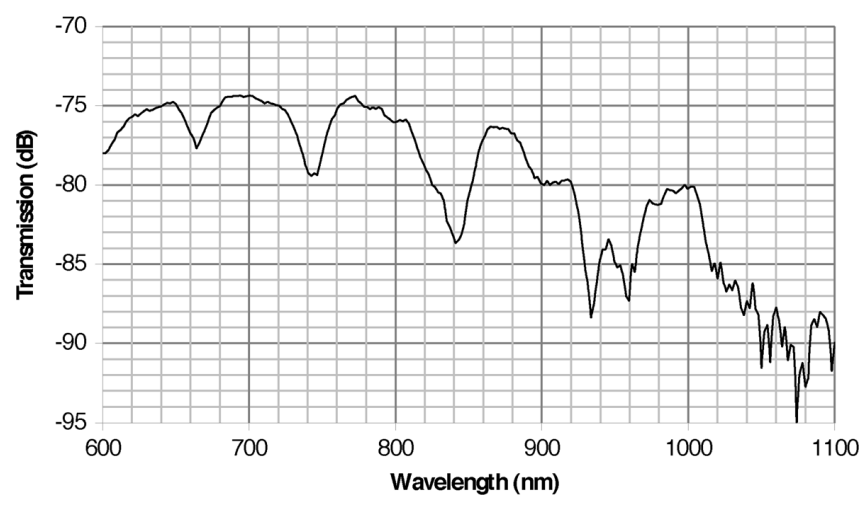

Fig. 3. Spectrum of LPG in $\mathrm{mPOF}$.

ization of residual polymer which increases the polymer concentration in the irradiated region [12], [13].

The long period grating fabricated was $2 \mathrm{~cm}$ in length and had a period of $1 \mathrm{~mm}$. The spectrum was measured using an optical spectrum analyzer (OSA) with minimum resolution of $50 \mathrm{pm}$, wavelength range between $350 \mathrm{~nm}$ and $1750 \mathrm{~nm}$ and dynamic range of $90 \mathrm{dBm}$. A halogen lamp of $150 \mathrm{~W}$ was used as the light source in the experiments. Fig. 3 shows the spectrum of the grating measured with $5 \mathrm{~nm}$ resolution in the wavelength range 600 to $1100 \mathrm{~nm}$. The spectrum has six notches corresponding to the coupling between the core guided mode and different cladding modes. The resonance at $950 \mathrm{~nm}$ is split into two bands that might correspond to two polarization states.

\section{Spectral Response When Exposed to Water}

Polymethyl methacrylate can absorb water from the surrounding medium up to a content of $2 \mathrm{wt} \%$ and has a diffusion coefficient of $6.7 \times 10^{-9} \mathrm{~cm}^{2} / \mathrm{s}$ measured at $23.5^{\circ} \mathrm{C}$ [14]-[16]. Water absorption results in volumetric and refraction index changes, as a consequence, Bragg gratings and long period gratings in fibers made with this polymer are good candidates for water detection without need of recoating the fiber with absorbent materials or filling with water the holes of microstructured fibers.

The response of the device to water is divided into two parts: the first one is the diffusion into the fiber, the second is the drying process of the fiber.

The mPOF LPG was immersed in a bath of distilled water and the grating spectrum was measured for $8.5 \mathrm{~h}$ until the diffusion of water into the fiber reached a stationary state. We show in Fig. 4 the wavelength shift of the resonance initially situated at $747 \mathrm{~nm}$ in Fig. 3.

We propose the following explanation for this behavior using (2), where in this case $X$ represents water content in the fiber. It should be understood that the effect of water absorption is to cause both an expansion of the material and an increase in refractive index [16]. We consider two aspects to the response. Upon submersion, water diffuses into the cladding region and initially has no effect on the core refractive index; consequently, (2) may be written

$$
\frac{\partial \lambda_{\mathrm{res}}}{\partial X}=\gamma \cdot \lambda_{\mathrm{res}} \cdot\left(\frac{1}{\Lambda} \frac{\partial \Lambda}{\partial X}-\frac{1}{\Delta n} \frac{\partial n_{\mathrm{cl}}}{\partial X}\right) .
$$

The sign of the wavelength shift therefore depends on the relative magnitude of the two terms in the brackets. In [17], the first 


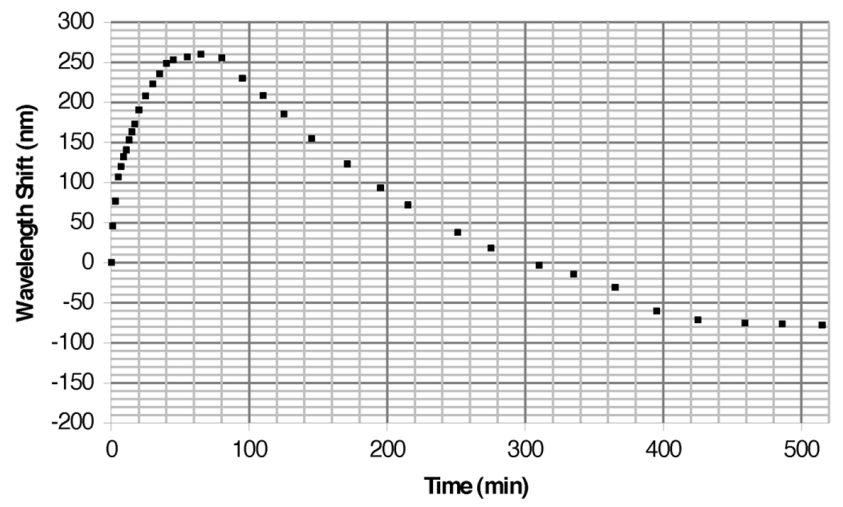

Fig. 4. Wavelength shift of a particular resonance ( $747 \mathrm{~nm}$ in Fig. 3) due to the diffusion of water into the fiber.
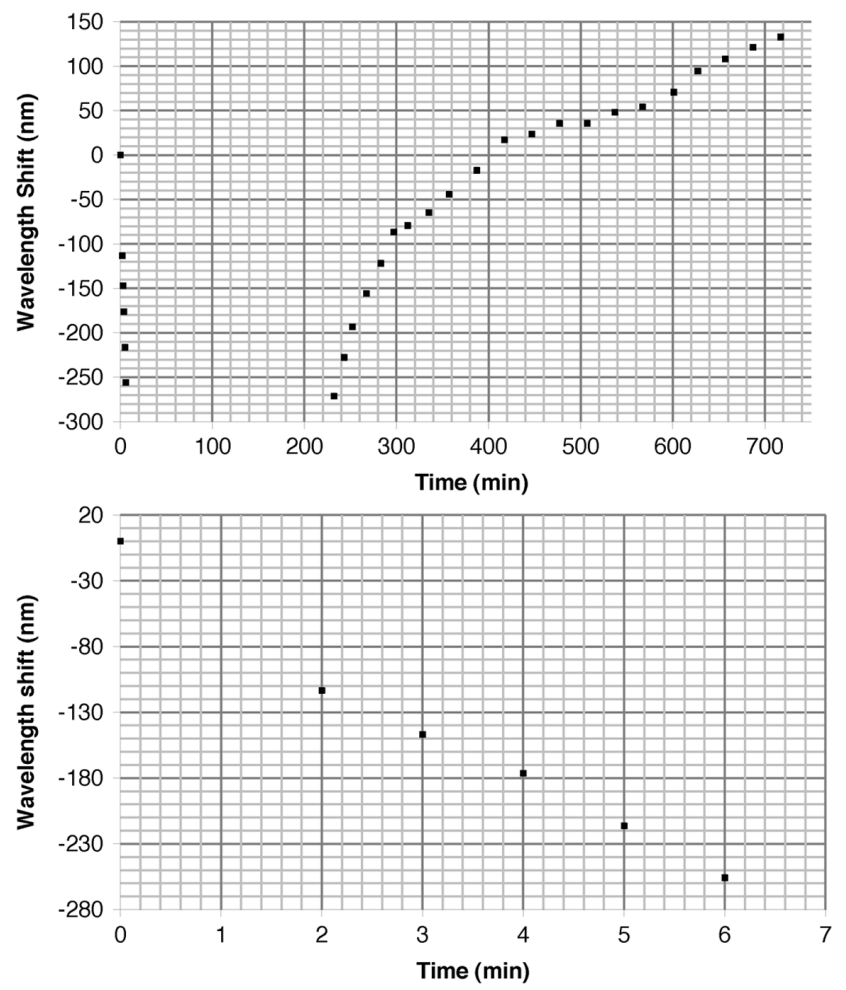

Fig. 5. Wavelength shift of a particular resonance (1070 nm in Fig. 3) when the fiber dries out. Top figure: long term evolution. Bottom figure: detail of the initial part of the process.

term, which describes swelling is measured at approximately $4 \times 10^{-3}$ for maximal water uptake, while the second term is approximately two orders of magnitude higher [18]. Consequently, the second term dominates and, since for this fiber $\gamma$ is negative [9], there is an initial positive wavelength shift.

When the water arrives in the core of the fiber, the water-induced change in the core index effectively cancels out the index change in the cladding. Though index cancellation may not be perfect due to the different nature of the core and the cladding, it makes the second term in the brackets within (2) negligible compared to the first term. As a result of this, the final steady state behavior is characterized by a negative wavelength shift from the dry value.

When the fiber is taken out of the water the polymer dries out and the opposite behavior is observed. Fig. 5 shows the drying

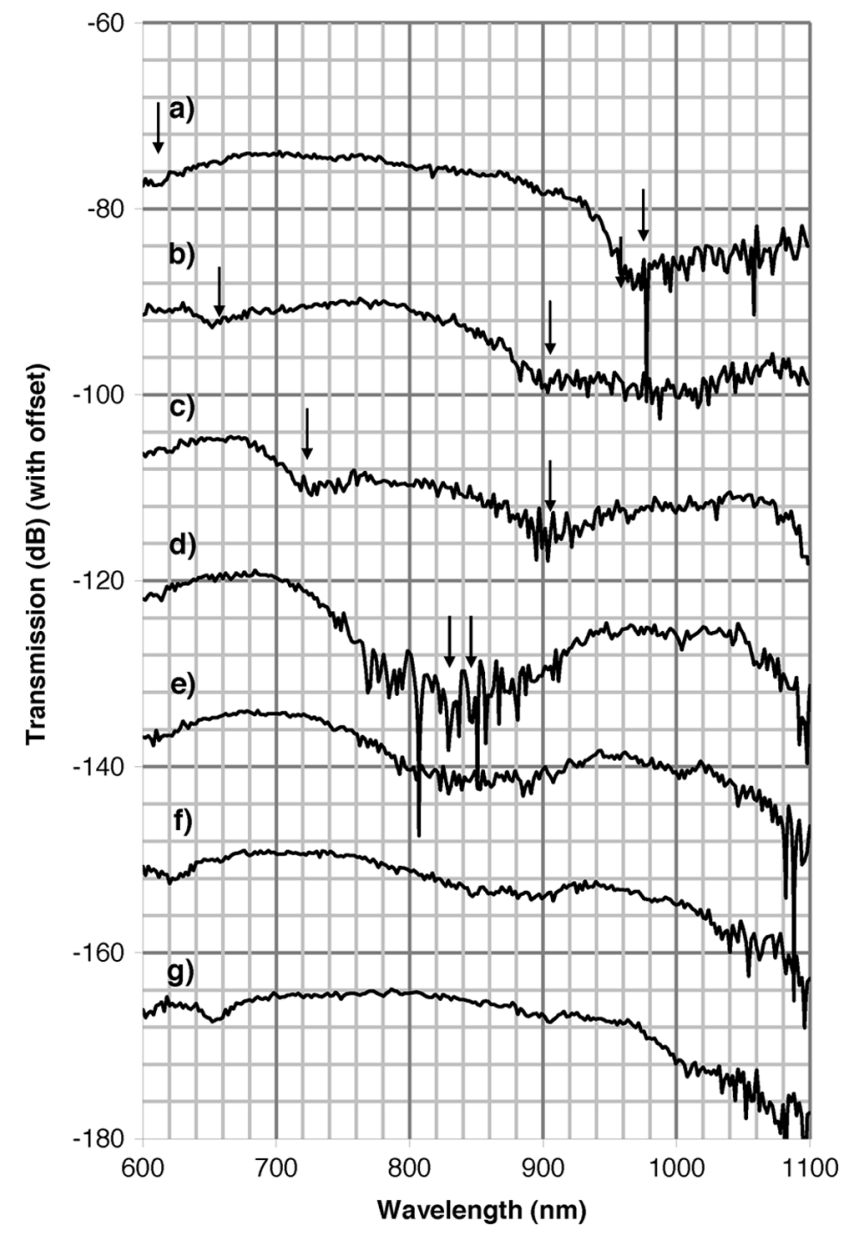

Fig. 6. Evolution of double resonance: (a) 19, (b) 21, (c) 23, (d) 24, (e) 25, (f) 26, and (g) $28 \mathrm{~min}$ after removal from water.

process using a different peak from that in Fig. 4, chosen to allow the largest negative wavelength shift to be observed (even so, the resonance under test was out of the wavelength measurement range during the time interval between 10 and $220 \mathrm{~min}$ ). Other bands show the same qualitative behavior. Initially there is a huge shift towards shorter wavelengths as water diffuses out of the cladding followed by a red shift when the core starts drying.

The spectrum was measured over $12 \mathrm{~h}$ and it did not reach the stationary state, indicating that some moisture remains in the fiber for a long time. After drying out the fiber by gentle heating $\left(<45^{\circ} \mathrm{C}\right)$ for $40 \mathrm{~min}$, the spectrum of Fig. 3 was recovered; it has not been investigated yet whether the drying time could be shortened. Experiments were repeated three times and no significant variations were observed.

It must be noticed the fast initial response of both processes (soaking and drying), wavelength shifts of $50 \mathrm{~nm}$ take place in the first $30 \mathrm{~s}$. LPGs response is much faster than Bragg gratings which have an activation delay of about $8 \mathrm{~min}$ in $\mathrm{mPOF}$ [16] because water takes time to reach the fiber core. This fast response makes LPGs in $\mathrm{mPOFs}$ suitable candidates for water level sensors.

Some modes of the grating spectrum exhibit a double resonance within the measurement range, with the core mode coupling energy to a cladding mode at two different wavelengths 
[11], [19], double resonances appear because of the specific dispersion curves of the core and cladding modes which make the resonance wavelength curve to have a turning point as function of the grating period for a specific set of modes in a range of periods. Since the resonance wavelength have opposite slopes as function of the grating period at both sides of the turning point, these resonances shift in opposite spectral directions when the properties of the waveguide are modified. As an example, Fig. 6 shows the evolution of a double resonance while the fiber dries out: two resonances were measured at 600 and $1100 \mathrm{~nm} 19 \mathrm{~min}$ after taking the fiber out of the water bath. As water leaves the fiber, these resonances move towards each other and five minutes later they coincide at $820 \mathrm{~nm}$, after which they gradually disappear.

\section{CONCLUSION}

In this paper, we have demonstrated what is to our knowledge the first LPG photoinscribed in microstructured polymer optical fiber using UV radiation. Furthermore the response of the LPG to water has been investigated and clearly reveals the water diffusion process at work. Results have direct application in the filed of optical fiber sensors as water level detectors and are a start point for relative humidity measurement.

\section{ACKNOWLEDGMENT}

D. Sáez acknowledges the comments of M. Andrés and assistance of FPU programs.

\section{REFERENCES}

[1] A. M. Vengsarkar, P. J. Lemaire, J. B. Judkins, V. Bhatia, T. Erdogan, and J. E. Sipe, "Long-period fiber gratings as band-rejection filters," $J$. Lightwave Technol., vol. 14, pp. 58-64, Jan. 1996.

[2] V. Bhatia and A. M. Vengsarkar, "Optical fiber long-period grating sensors," Opt. Lett., vol. 21, no. 9, pp. 692-694, 1996.

[3] H. J. Patrick, A. D. Kersey, and F. Bucholtz, "Analysis of the response of long period fiber gratings to the external index of refraction," $J$. Lightwave Technol., vol. 16, pp. 1606-1612, Sep. 1998.

[4] Y. Liu, L. Zhang, J. A. R. Williams, and I. Bennion, "Optical bend sensor based on measurement of resonance mode splitting of long-period fiber grating," IEEE Photon. Technol. Lett., vol. 12, pp. 531-533, May 2000.

[5] T. Venugopalan, T. Y. Leo, T. Sun, and K. T. V. Grattan, "LPG-based PVA coated sensor for relative humidity measurement," IEEE Sensors J., vol. 8, pp. 1093-1098, Jul. 2008.

[6] L. Rindorf and O. Bang, "Highly sensitive refractometer with photonic crystal-fiber long-period grating," Opt. Lett., vol. 33, pp. 563-565, 2008.

[7] S. H. Jung, D. H. Lee, K. H. Kwon, and J. W. Song, "Water sensor using macrobending-sensitive fiber for real-time submersion monitoring," Opt. Commun., vol. 260, pp. 105-108, 2006.

[8] K. R. Sohn, K. T. Kim, and J. W. Song, "Optical fiber sensor for water detection using a side-polished fiber coupler with a planar glassoverlay-waveguide," Sens. Actuators A, vol. 101, pp. 137-142, 2002.

[9] M. P. Hiscocks, M. A. van Eijkelenborg, A. Argyros, and M. C. J. Large, "Stable imprinting of long-period gratings in microstructured polymer optical fibre," Opt. Expr., vol. 14, pp. 4644-4649, 2006.

[10] M. Aressy, "Manufacturing optimisation and mechanical properties of polymer optical fibre," M.Phil. thesis, Birmingham Univ., Birmingham, U.K., 2006.

[11] X. Shu, L. Zhang, and I. Bennion, "Sensitivity characteristics of longperiod fibre gratings," J. Lightwave Technol., vol. 20, pp. 255-266, 2002.

[12] H. Dobb, D. J. Webb, K. Kalli, A. Argyros, M. C. J. Large, and M. A. Eikelenborg, "Continuous wave ultraviolet light-induced fiber Bragg gratings in few- and single-mode microstructured polymer optical fibers," Opt. Lett., vol. 30, pp. 3296-3298, 2005.
[13] M. J. Bowden, E. A. Chandross, and I. P. Kaminow, "Mechanism of the photoinduced refractive index in polymethyl methacrylate," Appl. Opt., vol. 13, no. 1, pp. 112-117, 1974.

[14] F. Beuche, "Diffusion of water in polymethyl methacrylate," J. Polymer Sci., vol. 14 , no. 16 , pp. 414-416, 1954.

[15] D. T. Turner, "Polymethyl methacrylate plus water: Sorption kineticks and volumetric changes," Polymer, vol. 23, pp. 197-2002, 1982.

[16] N. G. Harbach, "Fiber Bragg gratings in polymer optical fibers," M.S. thesis, Ecole Polytechnique Federale De Lausanne, Lausanne, Switzerland, 2008.

[17] S. H. Goods, R. M. Watson, and M. Yi, Thermal expansion and hydration behavior of PMMA molding materials for LIGA applications Sandia Nat. Labs, Tech. Rep. . SAND rep. SAND2003-8000, 2003.

[18] T. Watanabe, N. Ooba, S. Hayashida, T. Kurihara, and S. Imamura, "Polymeric optical waveguide circuits formed using silicone resin," $J$. Lightwave Technol., vol. 16, pp. 1049-1055, 1998.

[19] X. Shu, X. Zhu, S. Jiang, W. Shi, and D. Huang, "High sensitivity of dual resonant peaks of long-period fibre grating to surrounding refractive index changes," Electron. Lett., vol. 35, pp. 1580-1581, 1999.

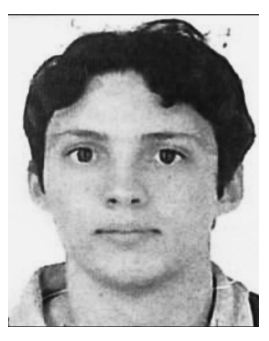

D. Sáez-Rodŕiguez graduated with a Degree from the University of Valencia, Valencia, Spain, in 2005. $\mathrm{He}$ is currently working towards the Ph.D. degree in photonics at the University of Valencia.

He works on inscription of gratings and your applications on fiber laser.

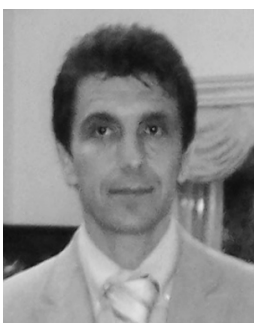

J. L. Cruz was born in Cuenca, Spain, in 1964. He received the $\mathrm{Ph}$.D. degree in physics from the University of Valencia, Valencia, Spain, in 1992.

Initially, his career focused on microwave devices for radar applications, afterwards, he worked for two years in optical fiber fabrication at the Optoelectronics Research Center, University of Southampton (U.K.). He is currently the Director of the Department of Applied Physics, University of Valencia, where he is conducting research on fiber lasers and amplifiers, photonic crystal fibers, fiber gratings, microwave photonics and sensors. He is co-inventor of six patents related with the above mentioned topics, author of about 100 publications in international journals and more than 130 papers in conferences.

Dr. Cruz is a member of Optical Society of Amera (OSA). He regularly acts as a reviewer of the IEEE and OSA journals, institutions for whom he has reviewed more than 60 manuscripts.

I. Johnson, photograph and biography not available at the time of publication.

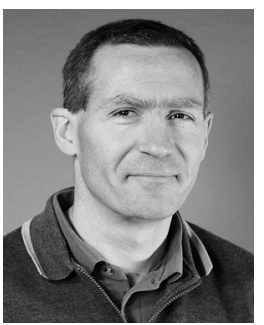

D. J. Webb received the B.A. degree in physics from the University of Oxford, Oxford, U.K., in 1983 and the Ph.D. degree in physics from the University of Kent, Kent, U.K., in 1989

He was appointed Reader in the Photonics Research Group, Aston University, in April 2001. Before then he was for ten years at the University of Kent and has also worked at Oxford University and for GEC Research. His main interests cover optical fiber sensing using in-fiber Bragg gratings and fiber interferometers and he has also published work on stimulated Brillouin scattering and photorefractive nonlinear optics. Current research involves medical and biochemical applications of optical sensing technology and the development of polymer optical fiber-based grating devices. $\mathrm{He}$ has published over 250 journal and conference papers on these subjects. 
M. C. J. Large received the B.Sc. degree from the University of Sydney, Sydney, Australia, and the Ph.D. degree in physics from Trinity College, Dublin, Ireland.

Following postdoctoral fellowships in France, she was a Lecturer at the Dublin Institute of Technology before returning to the University of Sydney in 2001. She is currently a Senior Research Fellow there, within the School of Physics.
A. Argyros graduated with a Degree from the University of Sydney, Sydney, Australia, in 2001 and received the Ph.D. degree in physics from the Optical Fibre Technology Centre, University of Sydney, in 2006.

$\mathrm{He}$ is a Senior Research Fellow at the Institute of Photonics and Optical Science (IPOS), School of Physics, University of Sydney, Australia. He works on microstructured polymer optical fibers and $\mathrm{THz}$ waveguides. 